\title{
Evaluation of antibody response of sheep to foot-and-mouth disease vaccine prepared by using different Montanide ${ }^{\mathrm{TM}}$ oil adjuvants
}

\author{
Çokçalışkan, C. ${ }^{1}$, Türkoğlu, T. ${ }^{1}$, Tuncer-Göktuna, P. $^{1}$, Sareyyüpoğlu, B. ${ }^{1}$, Aras-Uzun, E. ${ }^{1}$, Gündüzalp, C. ${ }^{1}$, \\ Kürkçü, A. ${ }^{1}$, Kara, $0 .{ }^{1}$, Karakaya, M. ${ }^{1}$, Gülyaz, V. ${ }^{2}$
}

${ }^{1}$ Republic of Turkey, Ministry of Agriculture and Forestry, Institute of Foot and Mouth Disease (SAP), Ankara, Turkey

${ }^{2}$ Harran University, Faculty of Veterinary Medicine, Virology Department, Şanlıurfa, Turkey

*Corresponding author: cancokcaliskan@gmail.com; can.cokcaliskan@tarimorman.gov.tr

\section{ARTICLE HISTORY}

Received: 4 September 2020

Revised: 8 January 2021

Accepted: 11 January 2021

Published: 25 March 2021

\begin{abstract}
Despite the widespread use of the conventional inactivated foot-and-mouth disease (FMD) vaccine, its immunogenicity is poor and the duration of its protection is short. In this study, humoral response to commercial ready-to-use Montanide ${ }^{T M}$ ISA 201 VG and Montanide ${ }^{T M}$ ISA 61 VG oil adjuvants and a common adjuvant Montanide TM ISA 206 VG developed by Seppic Inc., were evaluated for FMD antigens in sheep and double oil emulsion (w/o/w) formulations of Montanide TM ISA 201 and 206 and single oil emulsion (w/o) of Montanide ${ }^{\mathrm{TM}}$ ISA 61 have been prepared by using current FMDV antigens (O/TUR/07, A/ASIA/G-VII, A/TUR/16 and ASIA/ TUR/15). The animals $(n=48)$ were vaccinated subcutaneously with formulations and five sheep were maintained as an unvaccinated control group. Blood samples were taken at day $0,7,14,21,28,60,90,120$ and 150. Virus neutralization and liquid phase blocking ELISA tests were used to compare antibody response to vaccines prepared by using different Montanide ${ }^{\mathrm{TM}}$ mineral oils. The results showed that vaccines prepared by using Montanide ${ }^{\mathrm{TM}}$ ISA 61 and 201 gave better antibody response to FMD antigens than Montanide ${ }^{T M}$ ISA 206 formulation, although results were not statistically significant for certain days of sampling. Moreover, the overall type $\mathrm{O}$ antibody response of Montanide ${ }^{\mathrm{TM}}$ ISA 201 was found to be superior to Montanide ${ }^{\mathrm{TM}}$ ISA 61.
\end{abstract}

Keywords: Foot-and-mouth disease; Vaccine; Sheep; Virus neutralization; Adjuvant.

\section{INTRODUCTION}

Foot-and-mouth disease (FMD) is a highly contagious viral disease of cloven-hooved animals. (Diaz-San Segundo et al., 2017). FMD causes severe loss of productivity and affected countries suffer economically due to restrictions in international trade. The lesions are mild in sheep compared to cattle and pigs, and are mostly located at the interdigital cleft of feet. Lambs mostly die because of heart failure as a result of acute infection (Ryan et al., 2008).

FMD vaccines are prepared by using an inactivated whole virion, which is a weak immunogen (Doel, 1999). Hence repeated vaccine administrations are needed for effective control of the disease (Diaz-San Segundo et al., 2017). The disease has been eradicated in many countries by intense and widespread vaccination. Potent vaccines are essential prerequisite in the absence of other effective control measures (de Los Santos et al., 2018).

Until now, two different adjuvants have been mainly used in FMD vaccines: aluminum gels and mineral oil emulsions (Doel, 1999). Oil adjuvants slow the release of antigens from emulsions, known as the depot effect (lyer et al., 2001; Li et al., 2013). Vaccines can be prepared as a single or double oil emulsion by using mineral oils. Water in oil formulations are better at inducing an immune response than all other emulsion types because the antigen is constantly released from the oil phase. However, this emulsion type causes granulomas and pain at the injection site (Bonam et al., 2017). Commercial Montanide ${ }^{\mathrm{TM}}$ ISA formulations are commonly used in FMD vaccines. The most well-known is Montanide TM ISA 206 (ISA-206), which has a low viscosity, and high stability and induces adequate potency (Doel \& Pullen, 1990). The adjuvant relatively elicits good immunity in cattle with long-term duration (Barnett and Cox, 1999). Moreover, it was proposed that ISA 206 does not lead to viral protein-1 (VP1) proteolysis, which occurs in FMD vaccines with an aluminum adjuvant (Doel \& Pullen, 1990; Patil et al., 2002). Seppic Inc. developed Montanide ${ }^{\text {TM }}$ ISA 201 (ISA-201) as a new version of ISA-206 in order to improve the cellular immune response (Li et al., 2013). Similarly, Montanide ${ }^{T M}$ ISA 61 VG (ISA-61) was developed by the same company in place of Montanide ${ }^{\mathrm{TM}}$ ISA 50 for water in oil emulsions. This mineral oil provides an easy administration with stable, elongated and stronger immune response. It was especially recommended for weak immunogenic antigens (lyer et al., 2001). 
Generally, sheep are not included in the FMD vaccination programs in developing countries. That could be one of the reasons that there are few adjuvant studies on sheep (Patil et al., 2002; Selim et al., 2010). Another reason could be the similarity of the immune responses of sheep and cattle. On the other hand, sheep are an important source of income for people in many parts of the world and play an undeniable role in disease epidemiology (Braun et al., 2018). The mild nature of disease in the species can make the diagnosis difficult and facilitate the spread of infection. More than $90 \%$ of the positive sheep samples submitted to the World Reference Laboratory are diagnosed as FMDV serotype $O$ (Kitching \& Hughes, 2002). This fact stresses the necessity of tailoring an FMD vaccine specifically for sheep.

This study aimed to evaluate the antibody response in sheep to the vaccines prepared with different oil adjuvants, those that contribute to combat the disease efficiently.

\section{MATERIALS AND METHODS}

\section{Animals}

Fifty-three FMDV antibody seronegative, 8-month old male merino sheep obtained from a state farm were used. The animals were kept in barns with paddocks and fed ad-libitum during the experiment. The study was conducted according to EU directive 2010/63EU for animal experiments and under the authorization of the local animal ethics committee (24.01.16/03-3).

\section{Vaccines, Immunizations, and Sampling}

Montanide $^{\text {TM }}$ ISA 61, 201 and 206 adjuvants were obtained from Seppic SA (Paris, France) in $1 \mathrm{~L}$ bottles. BEl inactivated FMDV antigens, O/TUR/07, A/ASIA/G-VII, A/TUR/16 and ASIA/ TUR/15 were used to prepare the tetravalent vaccine formulations. The vaccine formulations with ISA-201 and ISA206 were water-in-oil-in-water (w/o/w) double emulsions, and the formulation with ISA-61 was a water-in-oil (w/o) single emulsion. In accordance with the manufacturer's recommendations, the antigen phase/adjuvant phase ratio was 50:50 (w/w) in double emulsion formulations, and 40:60 $(w / w)$ in the single emulsion formulation. Antigen amount per dose was the same for all formulations.

The blending was made in a beaker using a laboratoryscale propeller with four blades at a temperature of $30^{\circ} \mathrm{C}$ $\left( \pm 1^{\circ} \mathrm{C}\right)$. The mixing speed was $400 \mathrm{rpm}$ for double emulsions and $2,000 \mathrm{rpm}$ for the single emulsion (w/o). All formulations were mixed for 10 minutes and kept at $4^{\circ} \mathrm{C}$ overnight. The formulations were used after the confirmation of the emulsion formation by drop test (Flies \& Chen 2003).

Animals were divided into four groups, one unvaccinated control group consisting of 5 animals and three vaccinated groups consisting of 16 animals for each formulation.

The vaccines were administered subcutaneously at the rear part of the front leg of the animals. Blood samples were taken on days $0,7,14,21,28,60,90,120$ and 150 postvaccination $(p v)$.

\section{Virus Neutralization Test (VNT)}

Sera samples were inactivated in a water bath at $56^{\circ} \mathrm{C}$ for 30 minutes. Serial dilutions in $50 \mu$ quantity from 1:4 up to $1: 512$ were made on microplates by a multichannel pipetting robot (Intregra Viaflo Assist, Integra-Biosciences, Switzerland) to ensure reproducibility of dilution. Then, 50 $\mu \mathrm{l} 100 \mathrm{TCID}_{50}$ homologous viruses were added to the wells and incubated at $37^{\circ} \mathrm{C}$ in a $\mathrm{CO}_{2}$ incubator for one hour. Following incubation, $50 \mu \mathrm{l}\left(6 \times 10^{5}\right.$ per $\left.\mathrm{ml}\right)$ BHK-21 cells were added to the wells. After 48 hours of incubation at $37^{\circ} \mathrm{C}$ in a
$\mathrm{CO}_{2}$ environment the plates were stained with crystal violet and examined for cytopathic effect (CPE). Virus neutralization antibody titers, which block $100 \mathrm{TCI}_{50}$ viruses in $50 \%$ of the wells, were determined according to OIE manual (OIE, 2011).

\section{Liquid Phase Blocking ELISA (LPB-ELISA)}

The assay was carried out according to the method described by Hamblin et al. (1986). On the first day of the assay, 96-well ELISA plates were coated by polyclonal rabbit anti-FMDV O/TUR/07, A/ASIA/G-VII and ASIA/TUR/15 antibodies (the polyclonal sera produced in-house). The test and control sera were diluted twofold from a starting dilution of 1:16 in PBS in another U-bottom plate. The homologous antigen purified in sucrose density gradient was added to the diluted sera samples. Plates were stored at $+4^{\circ} \mathrm{C}$ overnight. On the second day, the ELISA plates were washed three times with PBS and $50 \mu \mathrm{l}$ antigen/antibody mixtures were transferred to ELISA plates and incubated for one hour at $37^{\circ} \mathrm{C}$. The plates were washed and homologous guinea pig anti-FMDV antibodies (the polyclonal sera produced in-house) were added. After one hour of incubation, polyclonal rabbit antiguinea pig IgG-HRP conjugate (Dako, P0141, Agilent CA, USA) was added and the same incubation conditions were repeated.

The plates were washed with PBS and the chromogen substrate was added. After 15 minutes of incubation at room temperature, the reaction was stopped by adding $1.25 \mathrm{M}$ sulfuric acid to the wells. Color developments were read by a plate reader (Versamax, Molecular Devices, CA, USA) at 492 $\mathrm{nm}$ wavelength.

\section{Statistical Analyses}

Statistical analyses were done by an SPSS software. The independent t-test (Levene) was used to compare the differences between groups. When the variances were not homogenous, the Games-Howell post-hoc test were utilized for multiple comparisons. Differences were evaluated as significant when $\mathrm{p}<0.05$.

\section{RESULTS}

\section{VNT}

The VNT test results were given in Figures $1 a, b, c$, and $d$. Antibody response was detected in all ISA groups on day 7 following vaccination. Antibody titers increased gradually for the vaccine formulated with ISA-201 and reached a peak on day 120 post vaccination (PV) for type O. ISA-61 group showed higher type $O$ titer as compared to ISA-201 group only on day $14 \mathrm{PV}$. A similar response pattern was obtained for A/ASIA/G-VII and A/TUR/16 formulations. Higher titers were obtained with ISA-61 formulation following ISA-201 and ISA-206 for both A serotypes. The virus neutralization response to A/ASIA/G-VII of ISA 61 group was significantly higher for days 14 and 21 PV compared to ISA-201 and ISA-206 $(p<0.05)$. Similarly, neutralizing antibody titers to ISA-61 was higher than ISA-201 and ISA-206 for A/TUR/16 antigen on days 14 and 28 PV $(p<0.05)$. For Asia 1 serotype, the highest titers were obtained by ISA-61 formulation for day 14 PV. Titers to ISA-61 and 206 on day 120 PV were similar. Although it was not statistically significant, the responses to ISA-206 were the lowest between the formulations throughout the study for all antigen types. In the unvaccinated group no increase in titers was noticed.

\section{LPB-ELISA}

ELISA results were given in Figures $2 a, b$, and $c$. Increase in antibody titer was detected from day $14 \mathrm{PV}$ in all vaccinated 
a)

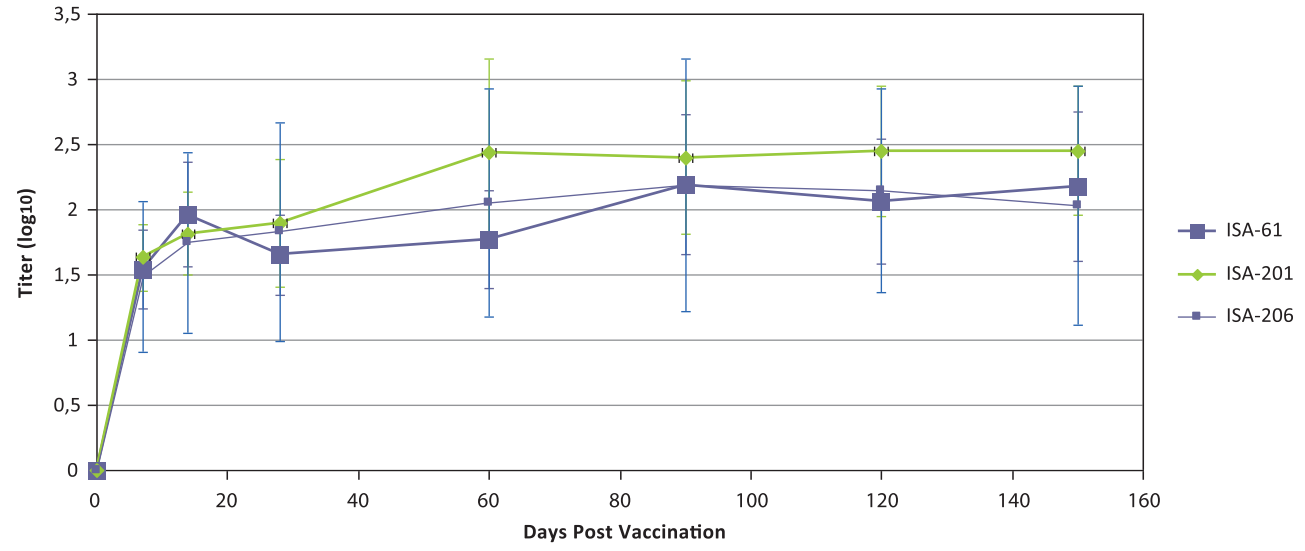

b)

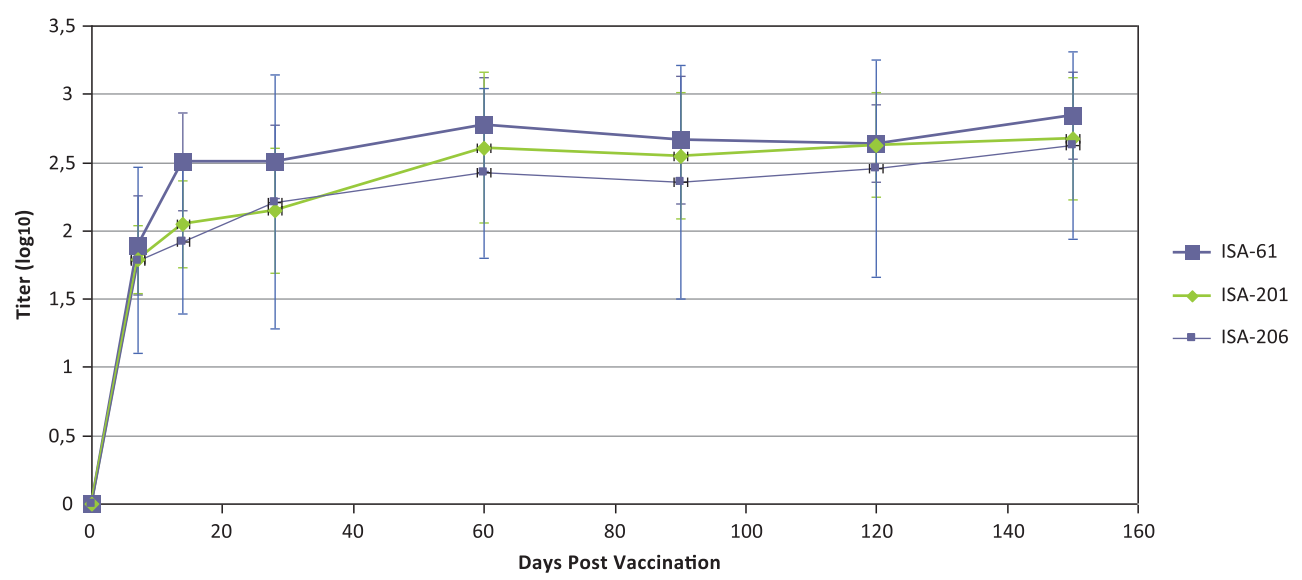

c)

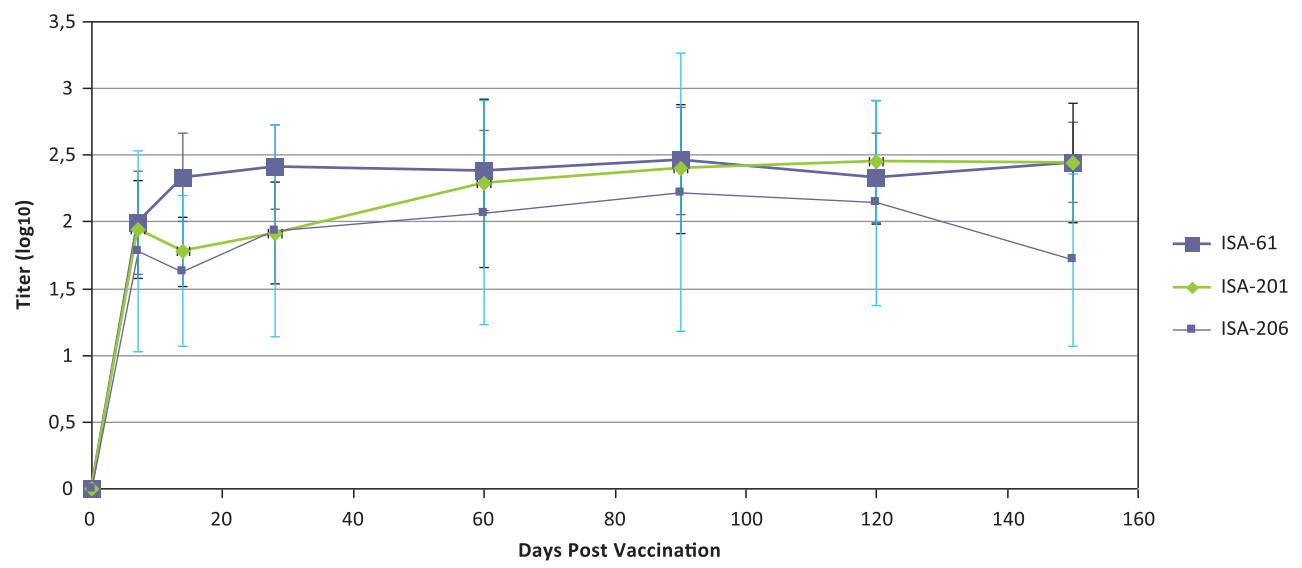

d)

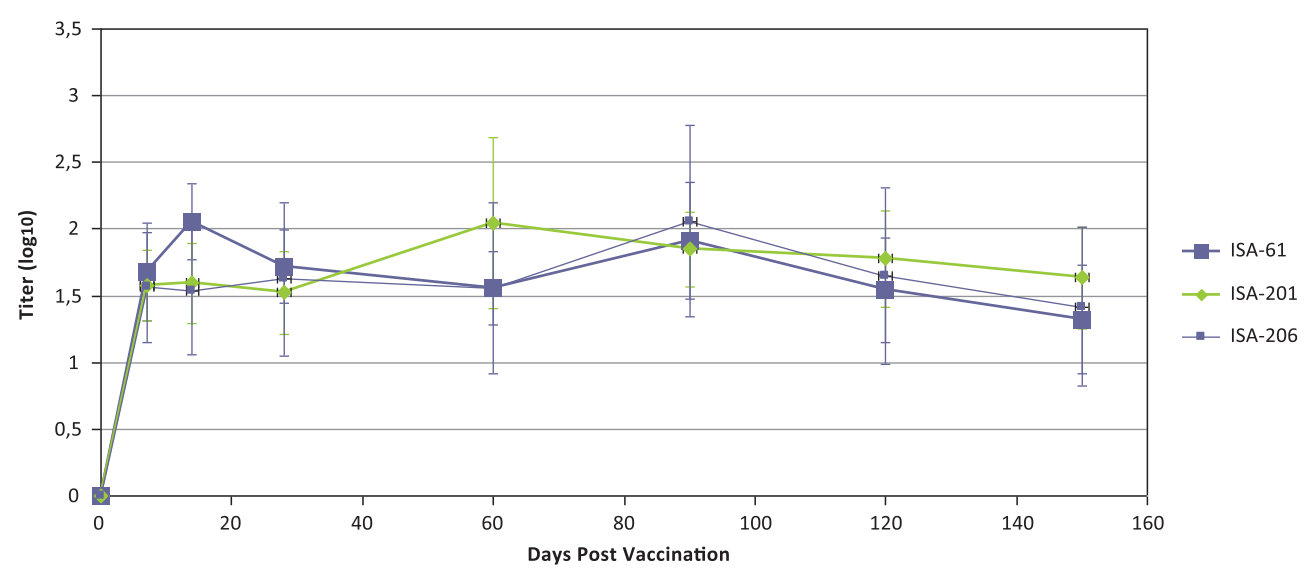

Figure 1. Comparison of FMD virus-neutralizing antibody titers in sera of sheep immunized with formulations prepared with different Montanide adjuvants.

a) O/TUR/O7, b) A/ASIA/G-VII, c) A/TUR/16, d) ASIA/TUR/15. 
a)

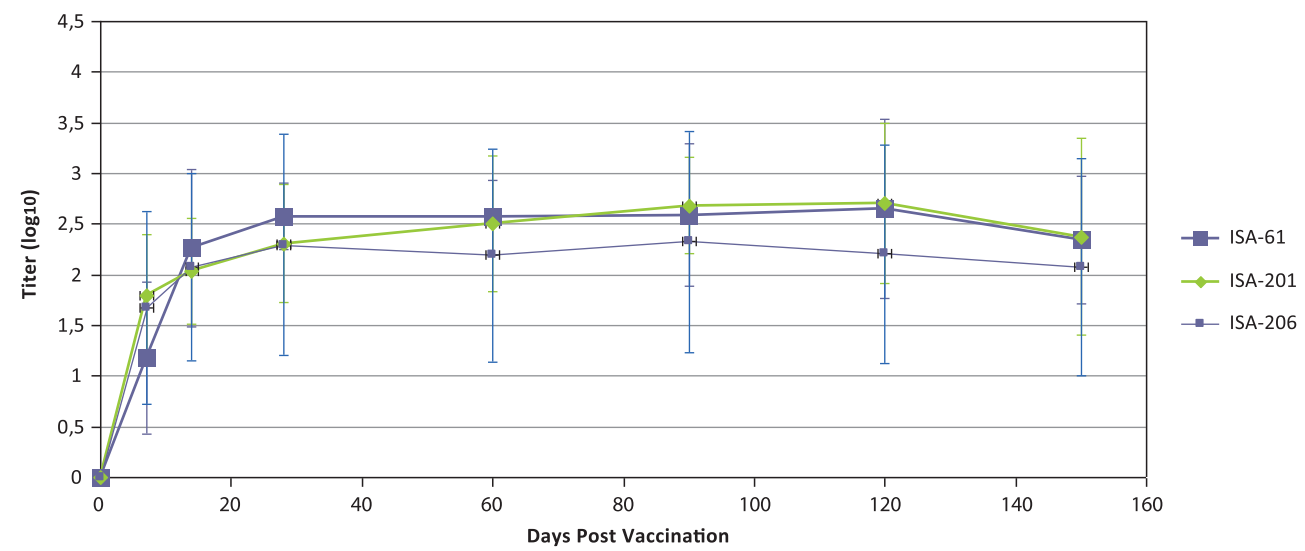

b)
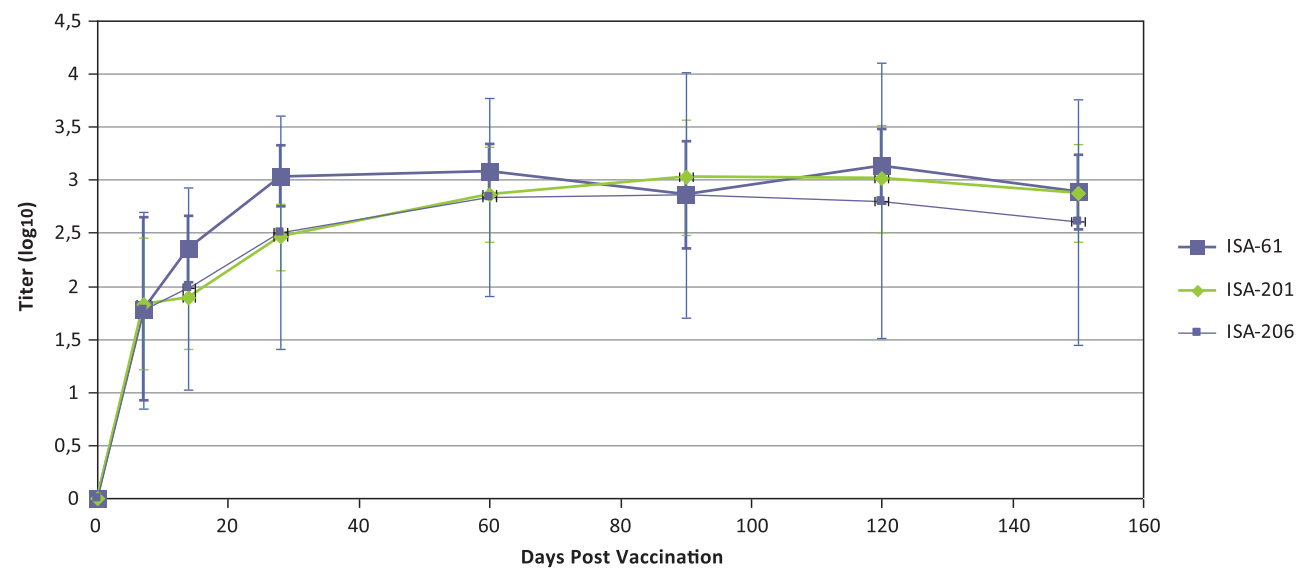

c)

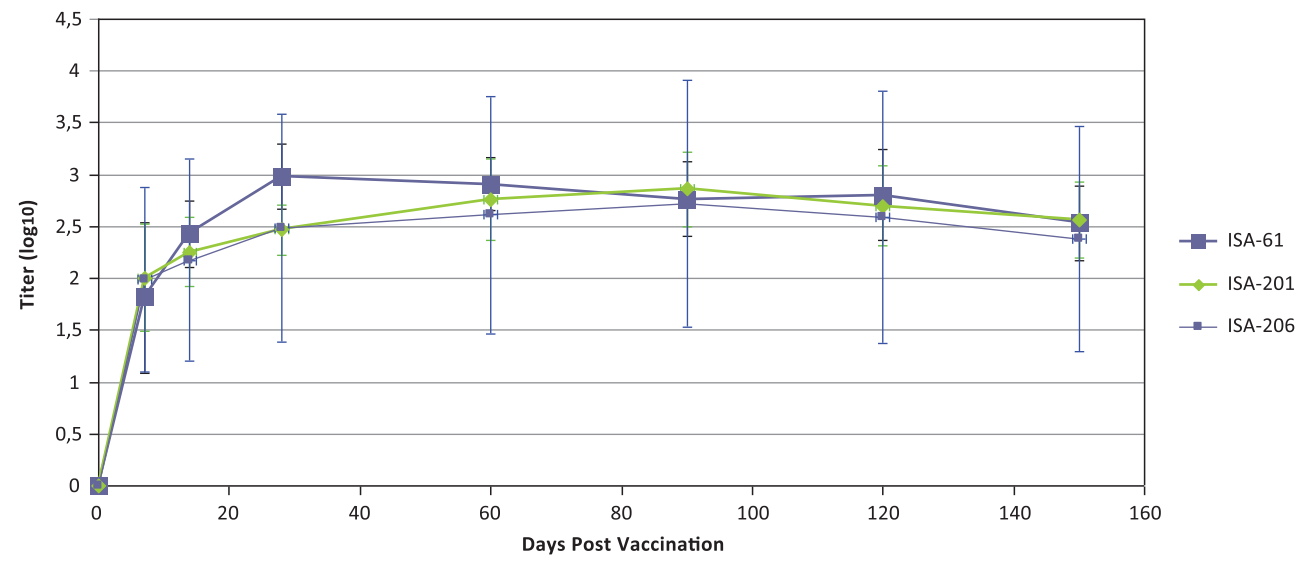

Figure 2. Comparison of FMD virus-specific total antibody titers observed in the LPB-ELISA from sera of sheep immunized with formulations prepared with different Montanide adjuvants.

a) O/TUR/O7, b) A/ASIA/G-VII, c) ASIA/TUR/15.

animals. The highest antibody levels were obtained by ISA61 formulation. For FMDV type 0 , day 7 pv antibody titer was higher for ISA-201 group compared to the other groups $(p<0.05)$. Antibody levels reached the peak on day 90 PV for all groups. Thereafter, FMDV type $O$ antibody titers of all vaccination groups started to decrease. Similarly, the highest antibody titers for FMDV type A were obtained by ISA-61 formulation from day $14 \mathrm{PV}$ to the end of the study. The differences are significant for days 14 and $28 \mathrm{pv}(\mathrm{p}<0.05)$. A dramatic decrease was detected after day 120 PV for type A with ISA-61 and 201. For Asia 1, the peak levels were reached by ISA-61 on day 28 PV, followed by ISA-201 $(p<0.05)$. The peak levels for ISA-201 were reached on day 60 PV. The lowest titers were obtained for ISA-206 group for days 14 and 28 PV $(p<0.05)$.

\section{DISCUSSION}

One of the most effective measures against FMD in endemic countries is vaccination. Despite the scientific progress, no strong alternative to the conventional inactive FMD vaccine has emerged in the field (Dar et al., 2013). The possibilities to increase the potency of the conventional vaccine are limited. One way of increasing the vaccine's potency is to 
increase antigen payload, which is effective up to some upper limit, and high cost and labor are also needed to produce the antigen (Doel, 1999). Another way of increasing its potency is to change or modify the adjuvant. Among those adjuvants, Montanide ${ }^{T M}$ ISA group have been safely and effectively used in livestock for a long time. One of them, ISA-206 has been used by various manufacturers. The attributes such as low viscosity, stability, safety and ease of administration were responsible for acceptance by the vaccine manufacturers. Moreover, antibody levels reached the protective level as early as 4 days in sheep after vaccination (Cox et al., 1999).

Different Montanide ${ }^{T M}$ formulations have been developed for various animal species in recent years. It was claimed that ISA-201 induced cellular immunity more strongly than ISA-206 and hence it was suggested that ISA-201 has the potential to replace ISA-206. The present study did not include $\mathrm{CMI}$ response analysis and the interpretations are based on the humoral immune response only. The higher antibody response both in terms of VNT and LPB-ELISA supports that claim indirectly. Dar et al. (2013) showed that ISA-201 gave a higher antibody response on day 7 PV when compared with ISA-206 in cattle. This higher response characterized by a high IgG2/IgG1 ratio and accompanied by a lymphoproliferative response continued till the end of that study. Similarly, in another study, neutralizing antibody responses were compared for ISA-201 and ISA-206 in pigs, and higher immune response was seen when ISA-201 was incorporated as an adjuvant (Park et al., 2014, 2016). Another adjuvant compared with ISA-206 was ISA-25, which forms water-in-oil emulsion. It was found that the efficacy and the stability of the vaccine formulated with ISA-25 were higher than ISA-206 (Barnett et al., 1996). lyer et al. (2001) studied ISA-50, ISA-57 and ISA-206 for FMD vaccines in the guinea pig model, and the highest antibody results were obtained by ISA-57, followed by ISA-50.

In an experiment carried out by Khorasani et al. (2016), ISA-61 formulation was compared with aluminum hydroxide and saponin formulations against FMD vaccine. The results demonstrated that the vaccine prepared by using ISA-61 was superior in terms of both neutralizing antibody titer and protective response $\left(\mathrm{PD}_{50}\right)$ (Khorasani et al., 2015). Likewise, in a study in cattle, gamma interferon levels were investigated for FMD vaccines prepared with ISA-206, 201 and 61 adjuvants. The highest gamma interferon level was obtained by ISA-61 formulation (Rizk et al., 2015). On the other hand, the researchers recommended ISA-201 instead of ISA-61 due to the lesions found in the injection site of ISA-61 (Gurung et al., 2014).

Since the highest early antibody response was obtained by ISA-61 formulation in this study, it could be concluded that our findings are in line with the earlier reports. In our study, no difference was found between the groups only in response to the FMDV type $\mathrm{O}$ antigen. This can be explained by the low stability of the type $O$ antigen in single oil emulsion. The same results were not seen in ELISA titers, except on the day $7 \mathrm{PV}$. The reason behind this is that the assay lacks the ability to differentiate between the integrity of antigens. Patil et al. (2002) proposed that although the type $O$ antigen is relatively more labile than the others, it still induced a strong immune response in sheep. This is due to the protective effect of the double oil emulsion of ISA206 against VP-1 hydrolysis. The double oil emulsion of ISA-201 might have protected the type $O$ antigen better than the ISA-61 formulation, as Patil et al. (2002) suggested. On the other hand, the epidemiologic importance of type 0 in sheep (Ryan et al., 2008) needs to be taken into account in the evaluation of the formulations. Besides, an infra- structural change in production plant is needed to produce vaccine with ISA-61 which demands high shear rate during the blending.

All literature findings indicated that formulations of single emulsions give better results than double emulsions (Bonam et al., 2017). However, our study demonstrated that the character of antigen also affects the antibody response. Although the best results have been obtained with the ISA61 formulation for the A and Asia 1 serotypes in our study, when other factors such as epidemiological importance of type $O$ for sheep and granuloma formation in muscles are taken into account, it was concluded that ISA-201 should be used as the alternative to ISA-206 in sheep for FMD vaccines.

\section{ACKNOWLEDGMENTS}

The authors wish to thank Abdullah Arslan, Ergün Uzunlu, Banu Bayri Özbilge and Ömer Şişman for their excellent technical help. Seppic, AirLiquide in Paris, France, supplied the sample Montanide ${ }^{T M}$ ISA adjuvants.

\section{Conflicts of Interest}

None.

\section{Financial Support}

This study was financially supported by The Republic of Turkey, Ministry of Agriculture and Forestry, General Directorate of Agricultural Research and Policy (Project Number: TAGEM/HSGYAD/16/A02/P02/69).

\section{REFERENCES}

Barnett, P.V. \& Cox, S.J. (1999). The role of small ruminants in the epidemiology and transmission of foot-and-mouth disease. Veterinary Journal 158(1): 6-13. https://doi.org/ 10.1053/tvjl.1998.0338

Barnett, P.V., Pullen, L., Williams, L. \& Doel, T.R. (1996). International bank for foot-and-mouth disease vaccine: assesment of Montanide TM ISA 25 and ISA 206, two commercially available oil adjuvants. Vaccine 14(13): 11871198. https://doi.org/10.1016/s0264-410x(96)00055-2

Bonam, S.R., Partidos, C.D., Kumar, S., Halmuthur, M. \& Muller, S. (2017). An Overview of Novel Adjuvants Designed for Improving Vaccine Efficacy. Trends in Pharmacological Sciences 38: 9. http://dx.doi.org/10.1016/j.tips.2017.06.002

Braun, R.O., Brunner, L., Wyler, K., Auray, G., García-Nicolás, O., Python, S., Zumkehr, B., Gaschen, V., Stoffel, M.H., Collin, N., Barnier-Quer, C., Bruggmann, R. \& Summerfield, A. (2018). System immunology-based identification of blood transcriptional modules correlating to antibody responses in sheep. NPJ Vaccines 3: 41. Doi:10.1038/s41541018-0078-0

Cox, S.J., Barnett, P.V., Dani, P. \& Salt, J.S. (1999). Emergency vaccination of sheep against foot-and-mouth disease: protection against disease and reduction in contact transmission. Vaccine 17: 1858-1868. https://doi.org/ 10.1016/s0264-410x(98)00486-1

Dar, P., Kalaivanana, R., Sieda, N., Mamoa, B., Kishorea, S., Suryanarayanad, V.V.S. \& Kondabattulaa, G. (2013). Montanide ${ }^{T M}$ ISA ${ }^{T M} 201$ adjuvanted FMD vaccine induces improved immune responses and protection in cattle. Vaccine 31: 3327-3332. http://dx.doi.org/10.1016/j.vaccine. 2013.05.078.

de Los Santos, T., Diaz-San Segundo, F. \& Rodriguez, L.L. (2018). The need for improved vaccines against foot-and-mouth disease. Current Opinion in Virology 29: 16-25. https://doi. org/10.1016/j.coviro.2018.02.005 
Diaz-San Segundo, F., Medina, G.N., Stenfeldt, C., Arzt, J. \& de Los Santos, T. (2017). Foot-and-mouth disease vaccines. Veterinary Microbiology 206: 102-112. http://dx.doi.org/ 10.1016/j.vetmic.2016.12.018

Doel, T.R. (1999). Optimization of the immune response to foot-and-mouth disease vaccines. Vaccine 17: 1767-1771. https://doi.org/10.1016/S0264-410X(98)00444-7

Doel, T.R. \& Pullen, L. (1990). International bank for foot-andmouth disease vaccine: stability studies with virus concentrates and vaccines prepared from them. Vaccine 8(5): 473-478. https://doi.org/10.1016/0264-410X(90)90249-L

Flies, D.B. \& Chen, L. (2003). A simple and rapid vortex method for preparing antigen/adjuvant emulsions for immunization. Journal of Immunological Methods 276(1-2): 239-242. https://doi.org/10.1016/S0022-1759(03)00081-4

Gurung, R.B., Purdie, A.C., Whittington, R.J. \& Begg, D.J. (2014). Cellular and humoral immune responses in sheep vaccinated with candidate antigens MAP2698c and MAP3567 from Mycobacterium avium subspecies paratuberculosis. Frontiers in Cellular and Infection Microbiology 4: 93. https://doi.org/10.3389/fcimb.2014.00093

Hamblin, C., Barnett, I.T., Hedger, R.S. (1986). A new enzymelinked immunosorbent assay (ELISA) for the detection of antibodies against foot-and-mouth disease virus. I. Development and method of ELISA. Journal of Immunological Methods 93(1): 115-21. https://doi.org/10.1016/00221759(86)90441-2

Iyer, A.V., Ghosh, S., Singh, S.N. \& Deshmukh, R.A. (2001). Evaluation of three 'ready to formulate' oil adjuvants for foot-and-mouth disease vaccine production. Vaccine 19: 1097-1105. https://doi.org/10.1016/S0264-410X(00)00337-6

Khorasani, A., Madadgar, O., Soleimanjahi, H., Keyvanfar, H. \& Mahravani, H. (2016). Evaluation of the efficacy of a new oil-based adjuvant ISA 61 VG FMD vaccine as a potential vaccine for cattle. Iranian Journal of Veterinary Research 17: 1, 54, 8-12.

Kitching, R.P. \& Hughes, G.J. (2002). Clinical variation in foot and mouth disease: sheep and goats. Revue Scientifýque et Technique Office International Des Epizooties 21(3): 505512.

Li, D., Zhou, C., She, D., Li, P., Sun, P., Bai, X., Chen, Y., Xie, B. \& Liu, Z. (2013). The comparison of the efficacy of swine FMD vaccine emulsified with oil adjuvant of ISA 201 VG or ISA 206 VG Journal of Biosciences and Medicines 1: 22-25. http://dx.doi.org/10.4236/jbm.2013.13005.

Park, M.E., Lee, S.Y., Kim, R.H., Ko, M.K., Lee, K.N., Kim, S.M., Kim, B.K., Lee J.S., Kim, B. \& Park J.H. (2014). Enhanced immune responses of foot-and-mouth disease vaccine using new oil/gel adjuvant mixtures in pigs and goats. Vaccine 32(40): 5221-5227. https://doi.org/10.1016/j.vaccine.2014.07.040

Park, M.E., Lee, S.Y., Kim, R.H., Ko, M.K., Park, J.N., Lee, K., Kim, S.M., Choi, J.H., You, S.H., Kim, B., Lee, J.S. \& Park, J.H. (2016). Altered adjuvant of foot-and-mouth disease vaccine improves immune response and protection from virus challenge. Trials in Vaccinology 5: 97-104. https://dx.doi.org/ 10.1016/j.trivac.2016.04.006

Patil, P.K., Bayry, J., Ramakrishna, C., Hugar, B., Misra, L.D., Prabhudas, K. \& Natarajan, C. (2002). Immune Responses of Sheep to Quadrivalent Double Emulsion Foot-andMouth Disease Vaccines: Rate of Development of Immunity and Variations among Other Ruminants. Journal of Clinical Microbiology 40: 4367-4371. https://doi.org/ 10.1128/jcm.40.11.4367-4371.2002
Rizk, S.A., El-Din, W.M.G., Mahdy, S.E., Ibrahim, E.S. \& Fakhry, H.M. (2015). Gamma Interferon Assay for Cellular Immune Response in Cattle Vaccinated With FMD Vaccine Adjuvanted with Different Montanide ${ }^{\mathrm{TM}}$ Oils. Global Journal of Medical Research: (G) Veterinary Science and Veterinary Medicine 15.

Ryan, E., Horsington, J., Durand, S., Brooks, H., Alexandersen, S., Brownlie, J. \& Zhang, Z. (2008). Foot-and-mouth disease virus infection in young lambs: Pathogenesis and tissue tropism. Veterinary Microbiology 127(3-4): 258-274. https:// doi.org/10.1016/j.vetmic.2007.08.029

Selim, A.M.A., Abouzeid, N.Z., Aggour, A.M. \& Sobhy, N.M. (2010). Comparative Study for Immune Efficacy of Two Different Adjuvants Bivalent FMD Vaccines in Sheep. Journal of American Science 6(10): 1292-1298.

World Organisation for Animal Health (OIE). (2011). Foot-andmouth disease, Chapter 8.5. In O. T. A. H. S. Commission (ed.), Terrestrial Animal Health Code $20^{\text {th }}$ ed. OIE World Organisation for Animal Health, Paris, France. 